\title{
Zwischen Moos und Wetterglas: Naturkundliche Interieurs und Objekte in der Literatur der deutschsprachigen Aufklärung
}

\author{
Jana Kittelmann ${ }^{1} \cdot$ Baptiste Baumann ${ }^{1}$
}

Published online: 6 June 2020

(c) The Author(s) 2020

\begin{abstract}
We investigate how objects of natural history, interiors, and practices of observation, collecting and experimenting as well as other forms of sensual perception of nature are represented and interpreted aesthetically in writings of the German-speaking Enlightenment. On the basis of selected examples, we analyze how systematizations and methods of natural history are transferred and adapted to literature and poetics.
\end{abstract}

Keywords Objects of natural history $\cdot$ Poetry $\cdot$ Literature $\cdot$ Enlightenment

\section{Einführung}

Das 18. Jahrhundert ist das Zeitalter der Erkundung und Systematisierung der Welt. Die von Naturforschern wie Carl von Linné, Albrecht von Haller oder George Louis Leclerc de Buffon betriebenen Klassifikations- und Systematisierungsbestrebungen (vgl. Lepenies 1988; Foucault 2015, S. 173ff.) beförderten das allgemeine Interesse an naturkundlichen Diskursen, Dingen und Verfahrensweisen. Forschungsreisen wie etwa Johann Jakob Scheuchzers Kartographierung der Alpen oder die globalen Unternehmungen Louis-Antoine Bougainvilles, James Cooks und Johann Reinhold und Georg Forsters, in deren Zuge nicht nur neue Erdteile entdeckt, sondern auch neue Arten wie im Rausch gesammelt, verzeichnet und bekannt wurden (vgl. Dawson 1976, S. 6), prägten das öffentliche Bewusstsein nachhaltig. Nicht zufällig hat Alexander von Humboldt retrospektiv in seinem geschichtlichen Überblick der „6 Epochen, welche als Hauptmomente die allmähliche Verbreitung der Erkenntnis vom Gedanken der Natur-Einheit" bezeichnen (Humboldt/Kohlrausch 2019, S. 210), die Entdeckungsreise der Forsters in eine Linie mit der „Erfindung neuer Organe zur Naturbeobachtung, das heißt Fernrohr, Wärmemesser, Barometer“

Jana Kittelmann

info@janakittelmann.de

1 Martin-Luther-Universität Halle-Wittenberg, Halle, Germany 
(ebd.) gestellt. Tatsächlich schlug sich die Entdeckung, Erforschung und technische Durchdringung der Natur in einer allgemeinen Neugierde, in einem verstärkten Interesse an naturkundlichen Objekten, Praktiken, Experimenten, Beobachtungs- und Sammlungsformen inklusive deren seit dem 17. Jahrhundert ständig perfektionierten Apparaturen, Instrumenten und Geräten wie Mikroskopen, Barometern, Botanisierschränken- und bestecken oder Herbarien nieder (vgl. u.a. Richter 1972, S. 199-202). Eine wissenschaftliche Betrachtung der Welt in all ihren Facetten war nicht mehr nur einem exklusiven Gelehrtenkreis vorbehalten, sondern auch Laien zugänglich. Von der damit verbundenen Popularität naturkundlich-epistemischer Interieurs und Praktiken zeugt etwa der Eintrag zu „Sammlung (Samen=), Samen-Kabinett“ in Krünitz' bekannter Oeconomischen Encyklopädie. Die Bauanleitung bezog sich dabei auf ein konkretes Gebrauchsmöbel des 18. Jahrhunderts: Den Samenschrank des Ratsmeisters und Gartenbau-Experten Christian Reichart (vgl. Stört 2018).

Naturwissenschaftliche Apparate als wichtige Gebrauchsgegenstände erfahren besonders um 1760 einen starken Popularisierungsschub. Innerhalb etwa eines Jahrzehnts erscheinen mehrere Schriften, die Instrumente zum privaten Gebrauch oder gar Bauanleitungen darstellen und erläutern. Führend sind hier Naturforscher wie Martin Frobenius Ledermüller, der seinen Mikroskopischen Gemüths- und Augen-Ergötzungen Abbildungen von Pflanzen, Tieren und verschiedenen Experimentiergeräten in hervorragender Qualität beifügte (Ledermüller 1762), ${ }^{1}$ oder der jüngere Jakob Franz von Bianchy, der 1762 Das Merkwürdigste vom Barometre und Thermometre publizierte (Bianchy 1762). ${ }^{2}$ Philosophen und Ästhetiker wie der in Preußen lebende Schweizer Johann Georg Sulzer, der zeitlebens auch als Naturforscher tätig war, bauen sogar selbst Thermometer und Barometer und berichten darüber in verschiedenen literarischen Medien, in Abhandlungen, Reiseberichten und Briefen (vgl. etwa Sulzer 1758). Auch in vornehmlich an ein jugendliches Publikum gerichteten Sammlungen und Kompendien wie dem Spectaculum Naturae \& Artium waren Ausführungen zu Barometer und Mikroskop inklusive ansprechender Abbildungen des renommierten Kupferstechers Johann Wilhelm Meil zu finden. (Abb. 1 und 2)

Die Schnittstellen zwischen Naturkunde, Philosophie und Ästhetik sind im 18. Jahrhundert zahlreich. Die für spätere Epochen charakteristische Grenzziehung zwischen Naturwissenschaft und Dichtung ist noch nicht so stark ausgeprägt. Naturkundliche Beobachtung, Systematisierung, technische und dichterische Aneignung der Natur schließen sich nicht aus (vgl. dazu Richter 1972, S. 11-18). Im Gegenteil: Wie Harro Segeberg formuliert hat, schrieb insbesondere die „Literatur der Aufklärung an der Erfolgsgeschichte einer Naturwissenschaft mit, die als experimentell-explikative Naturlehre wie als zur angewandten Mathematik gerechnete mechanisch-apparative Technologie gegen die Statik überlieferter Weltbilder das dynamische Prinzip einer technisch vermittelten autonomen Naturbetrachtung setzte“ (Segeberg 1997, S. 28, vgl. auch Stadler 2003, Forrer 2013). Neben der

\footnotetext{
1 Vgl. zum außerhalb der Wissenschaftsgeschichte kaum wahrgenommenen Ledermüller: Stadler 2003, S. $62 \mathrm{f}$.

2 Zur Tätigkeit Bianchys als Instrumentenbauer und -verkäufer, vgl. Hochadel 2003, S. 187ff.
} 


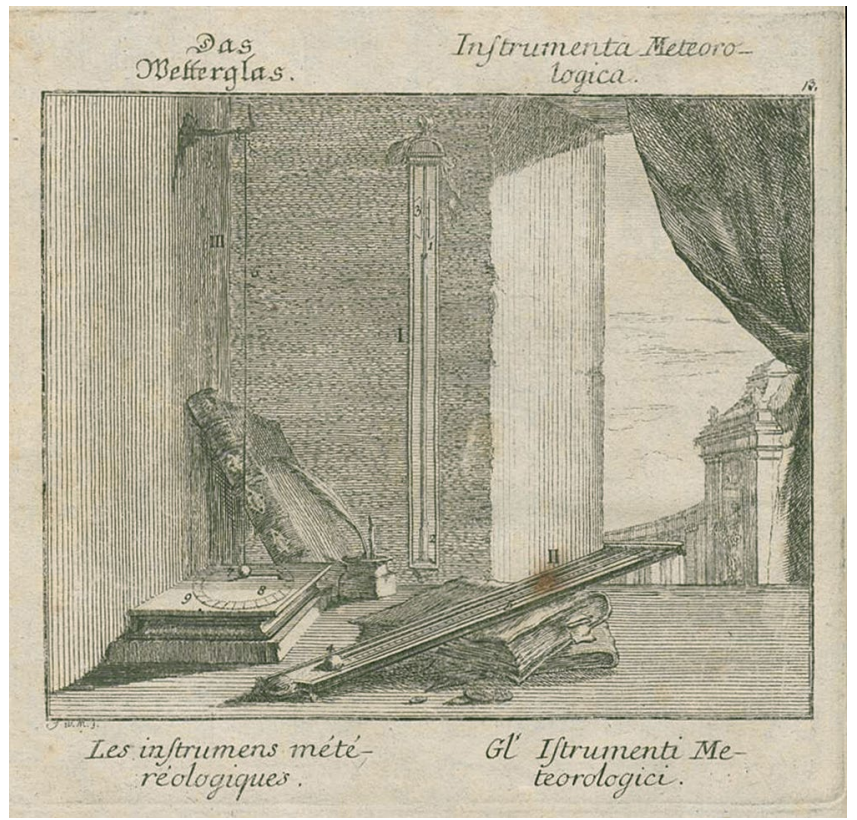

Abb. 1 Johann Wilhelm Meil: Wetterglas, in: Spectaculum Naturae \& Artium, Berlin 1761 (unpag.)

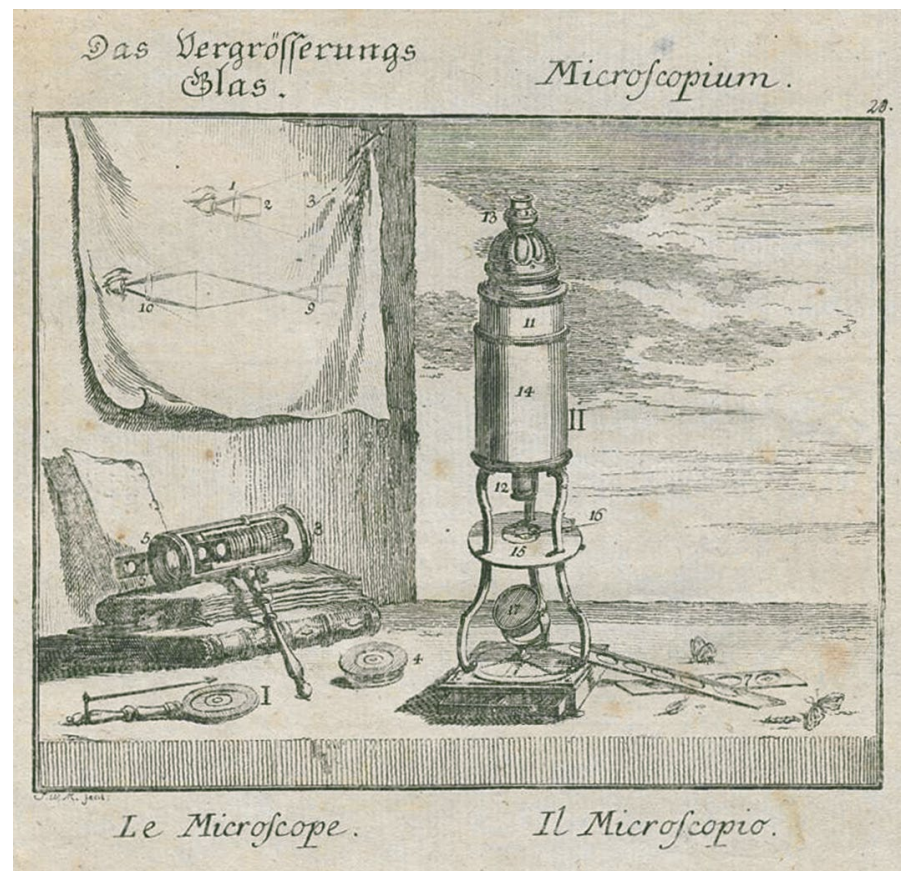

Abb. 2 Johann Wilhelm Meil: Mikroskop, in: Spectaculum Naturae \& Artium, Berlin 1761 (unpag.) 
Abb. 3 Johann Rudolf Schellenberg: Frontispiz zu Johann Heinrich Sulzer: Die Kennzeichen der Insekten, Zürich 1761

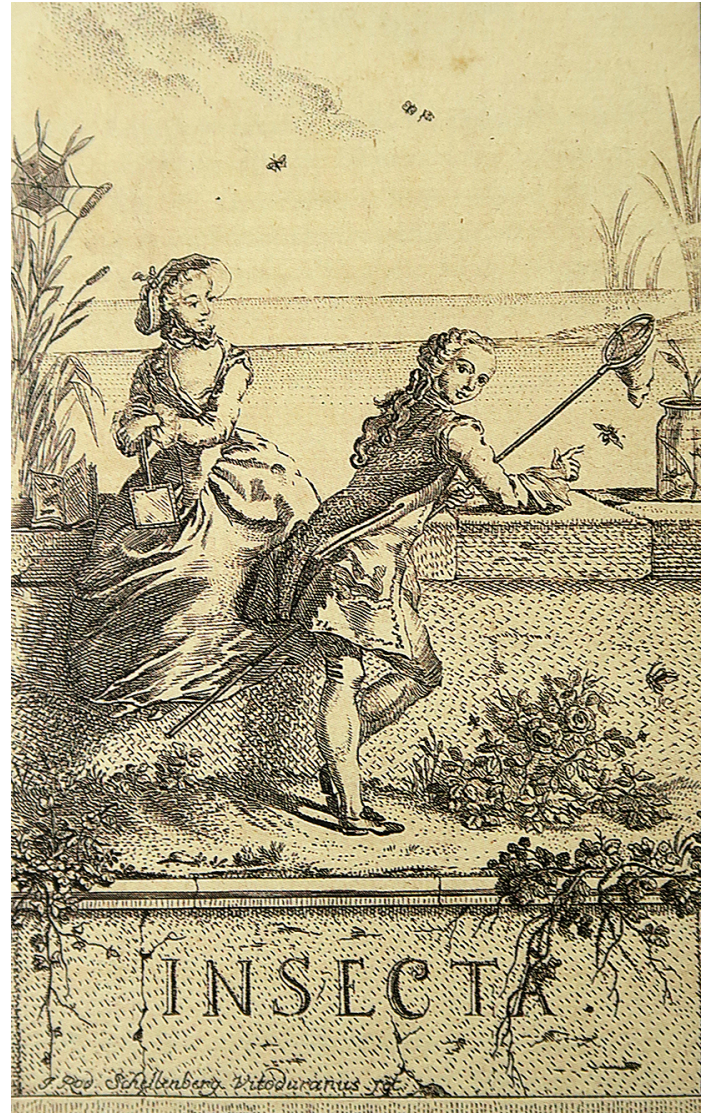

Popularisierung naturwissenschaftlicher Apparaturen trifft das auch und gerade für naturkundliche Praktiken zu (Abb. 3). Die Zahl der als Naturforscher tätigen Dichter, Philosophen und Publizisten der Aufklärung ist hoch. Insbesondere Botanisieren kommt unter Literaten und Philosophen in Mode und justiert auch die literarische Landschaft neu (vgl. Kittelmann 2018a). Bekannteste Beispiele dürften neben Albrecht von Hallers Die Alpen die botanischen Lehrbriefe Jeans-Jacques Rousseaus oder die Arbeiten des botanophilen Goethe sein, der nicht nur Gingkoblätter, Hyazinthen und Artischocken in Begleitung von Billets und Briefen an vertraute Korrespondenzpartner*innen übersandte, sondern seine botanischen Erkenntnisse auch in Fachpublikationen oder Lehrgedichten einem breiteren Publikum übermittelte. ${ }^{3}$ Auf der anderen Seite hegen in der philosophischen Naturlehre und deren Apparaturen und Praktiken gebildete Forscher und Gelehrte wie Albrecht von Haller, Johann Georg Sulzer, Abraham Gotthelf Kästner, Johann Georg Zimmermann, Johann Caspar Lavater um die Jahrhundertmitte und darüber hinaus zugleich

\footnotetext{
${ }^{3} \mathrm{Zu}$ Goethes breiten naturwissenschaftlichen Interessen und Tätigkeitsfeldern, die nicht nur die Botanik umfassten, vgl. Knebel et al. 2019.
} 
einen dichterischen Anspruch. Die zunehmende Thematisierung und literarische Überformung einer wissenschaftlichen Erfahrbarkeit von Naturereignissen und Naturphänomenen deuten schließlich auf einen verbreiteten Versuch hin, die Dichtung auch als einen Ort der fundierten Welt(er)kenntnis zu beanspruchen.

Der Präsenz von Objekten und Dingen (vgl. dazu Berndt/Fulda 2012) kommt dabei eine hohe Bedeutung zu, obgleich oder gerade weil ihnen auch in Texten des 18. Jahrhunderts das-wie es Dorothee Kimmich formuliert hat—,Entscheidende“ fehlt: ,,ihre Dinghaftigkeit, ihre Materialität.“ Im Gegensatz zu Naturalienkabinetten, Sammlungen oderMuseen sind,,Dingein Texten keinemmenschlichen Sinnunmittelbar zugänglich, sind weder fühlbar noch sichtbar, man kann sie weder hören noch riechen, sondern muss sie imaginieren." (Kimmich 2018, S. 21) Bei einer solchen Verschiebung von Wirklichkeitserfahrung durch Instrumente, Präparate oder abstrakte Zeichen, die als ,synchrone Problemlage“ (Segeberg 1997, S. 29f.) beschrieben werden kann, offenbaren sich durchaus unterschiedliche Zugangsweisen zur literarischen Umsetzung wissenschaftlicher Erkenntnis.

Hier will der Beitrag ansetzen und der Frage nachgehen, wie sich diese Imagination von konkreten naturkundlichen Dingen und damit verbunden die Praktiken der Beobachtung, des Sammelns, Experimentierens sowie andere Formen der sinnlichen Erfahrung in der textlichen Repräsentation darstellen (Hoffmann 2006, S. 43-45). Der Fokus richtet sich dabei auf eine bestimmte Autorengruppe (tatsächlich fast ausschließlich Männer) ${ }^{4}$ der Aufklärung, die als Naturforscher und Dichter tätig waren und sich somit an der Schnittstelle zwischen Naturhistorie, Poesie und Ästhetik bewegten. Anhand ausgewählter Beispiele soll untersucht werden, wie Ordnungen, Praktiken und Verfahrensweisen der Naturgeschichte in literarische und poetologische Verfahrensweisen übertragen werden und/oder mit ihnen korrespondieren. Inwiefern gewinnen dabei neben der freien Natur und der Landschaft auch konkrete Wissensräume wie der Garten oder das Naturalienkabinett an Bedeutung? Welche Formen der literarischen Inszenierung und ästhetischen Interpretation von naturkundlichem Material und Interieur eröffnen sich in den Texten?

Aufklärung markiert dabei eher einen fließenden und durchlässigen Epochenbegriff, der hier nicht eingrenzt, sondern verschiedene naturhistorische, dichterische, philosophische und anthropologische Strömungen, Denkansätze und Diskurse in sich vereint. Die ausgewählten Diskurse und Akteure sind facettenreich und als Impuls zu verstehen. Die Auswahl stützt sich jedoch auf eine historisch greifbare Denker-, Dichter- und Forschergemeinschaft, die nicht zuletzt als Gegenstand der Netzwerk- und Korrespondenzforschung ihre literarische bzw. literaturgeschichtliche Präsenz behaupten kann (vgl. u.a. Lütteken 2003; Stuber 2005; Décultot et al. 2018).

\footnotetext{
${ }^{4}$ Die Beschäftigung mit der Naturkunde war durchaus geschlechterübergreifend. Johann Georg Sulzer und seine Braut Wilhelmine Keusenhoff waren beispielsweise leidenschaftliche Insektensammler. Klopstock bewunderte Wilhelmines naturkundliche Sammlung, die fast den Charakter eines kleinen Naturalienkabinetts gehabt haben muss. In einem Brief an Maria Sophia Schmidt schwärmte er von ,Sulzers Braut, ein Mädchen mit schönen Augen u Verstande, die in ihrer Puzstube verschiedene Kästchens von raren Sachen hat, die zur Naturlehre gehören." (Klopstock 1979, S. 107) Ein Stich von Schellenberg zeigt ebenfalls ein Paar beim Fangen von Schmetterlingen und Insekten. Zudem war Botanisieren vor allem unter Frauen sehr populär.
} 


\section{Naturdingliche Dichtung}

Schon 1749 huldigte der preußische Dichter und Publizist Karl Wilhelm Ramler in seiner Ode Auf einen Granatapfel, der in Berlin zur Reife gekommen sowohl der Erkundung der Welt als auch der Kultivierung exotischer Sorten im märkischen Sand (Ramler 1767, S. 13f.):

Find' ich dich hier in deiner grünen Krone?

Zerspaltest du die purpurrothe Brust

An dieser Sonn'? o Liebling der Pomone!

O Proserpinens Apfel! die mit Lust

Und Wollust deine goldnen Körner

Im Reich des Höllengottes ass,

Und allen Nektar ferner

Und den Olymp vergass.

Der Erdball ändert sich: das Meer entfliehet,

Und macht dem Pfluge Raum; der Fels sinkt ein;

Und, o Berlin! dein dürrer Boden blühet:

Pomona füllt ihr Horn in dir allein,

In dir kann Flora, nach Begehren,

Sich tausendfache Kränze drehn,

Und ganz verdeckt in Aehren

Die blonde Ceres gehn.

Neben der unverkennbaren Intention Ramlers, Preußen unter der Regentschaft Friedrichs des Großen als Ort der üppigen Blüte und des fruchtbaren Aufschwungs zu kennzeichnen, ist bemerkenswert, dass ein Ereignis, von dem man annehmen könnte, dass es lediglich an der Naturkunde interessierte Gelehrte beschäftigt, breitenwirksam Furore machte und sogar zum Thema einer Ode avancierte. Ramler, der in Berlin einen eigenen Garten besa $\beta^{5}$, war dabei nicht nur an botanischen und pflanzenkundlichen Diskursen interessiert. In den von ihm gemeinsam mit Johann Georg Sulzer herausgegebenen Critischen Nachrichten aus dem Reiche der Gelehrsamkeit, die seit 1752 von Christlob Mylius redigiert wurden, stehen unter anderem Einträge zur Auffindung eines „Elephantengerippes“, zu neuen Entdeckungen auf Island, zu Waffen der Indianer und zu „Kieselsteinen“ neben Abhandlungen zu einem Florentinischen Thermometer, zu einem in der Nacht blühendem, „Traurigen Baum“ in Goa oder zur erfolgreichen Befruchtung einer Zwergdattelpalme in Berlin nebeneinander. Letzterer

\footnotetext{
5 Vgl. dazu Ramlers zahlreiche Beschreibungen seines Gartens in seinen Briefen an Johann Wilhelm Ludwig Gleim (Schüddekopf 1906/1907, u.a. Bd. 1, S. 46, 113). Auch Gelehrte wie Sulzer besaßen eigene Gärten mit Beeten, Arboreten und kleinen Alleen. Vgl. dazu u.a. Ramler an Gleim: „Man trift unsern Sulzer jetzt niemals in vier Wänden an [...] er pflantzt Tannen für die Nachwelt, und Cichorienwurtzeln für seine Gäste.“ (ebd., Bd. 2, S. 352). Sulzer selbst wollte nichts Geringeres als den Garten des Epikur wiederherstellen und „die Musen in dem schatten der orangen bäumen [...] loken.“ (Sulzer in einem Brief an Johann Jakob Bodmer, 30. März 1753, Zentralbibliothek Zürich, Ms Bodmer 5a).
} 
als Experimentum Berolinense in die Naturgeschichte eingegangener Versuch ${ }^{6}$ geht auf den Botaniker Johann Gottlieb Gleditsch zurück und regte u.a. Abraham Gotthelf Kästner zu dem Epigramm: Auf den Palmbaum, der 1749 im Garten der Königl. Preuß. Akademie der Wissenschaften reife Früchte trug an. Kästner, spezialisiert auf Lehrgedichte (vgl. dazu Baasner 1987; Baasner 1991), die nicht selten ironisch über neueste wissenschaftliche Erkenntnisse und naturkundliche Phänomene informierten, forderten zudem Apparaturen wie Das Wetterglas, ,[d]as mir von kalt und warm in sieben Zungen spricht" zu einem Epigramm heraus. Damit gesellte er sich zu Anna Louisa Karsch, die in ihrem Gedicht Die Sommer-Nässe, an Herrn Gleim dem Barometer (das heißt dem Wetterglas) ebenfalls prominenten Platz einräumt (Karsch 1764, S. 161):

Die schönen Kinder fühlen lange Weile,

Ihr Auge fragt das Wetter-Glas:

Ob bald die Sonne das Gewölk zertheile?

Den Tannenhäyn macht es zu naß.

Karschs, Kästners und Ramlers Gedichte sowie Beiträge in Lexika wie Krünitz' Enzyklopädie oder in Zeitschriften und Journalen, die zuweilen sogar Titel wie Patriotisches Wetterglas tragen, sind nur einige Beispiele, die auf die Präsenz von naturkundlichen Dingen, Objekten und Instrumenten in der poetischen und publizistischen Landschaft des 18. Jahrhunderts verweisen. Damit verbunden werfen sie zugleich die Frage danach auf, wie sich das auf die Materialität von bzw. die Materialitätsdiskurse in Texten der Aufklärung auswirkt. Wie beeinflussen technologische und naturhistorische Entwicklunge, Praktiken und Objekte die Form, die Wahr: löschennehmung, die Motivik von Texten, aber auch die Ausstattung und Illustrierung von Büchern? Inwiefern kann die professionell oder laienhaft betriebene wissenschaftliche Weltbetrachtung unter bestimmten Bedingungen auch als ein Format, eine Perspektive, ein Sujet der Literatur dienen?

\section{Lavaters Lupe und Kästners Fernrohr}

Im zweiten Band von Johann Caspar Lavaters Physiognomischen Fragmenten findet sich eine Vignette, die drei kindliche Figuren in ländlicher Szenerie zeigt (Abb. 4). Das Mädchen unter ihnen inspiziert mit der Lupe in der Hand einen Schmetterling. Einer der beiden Jungen versucht ein Insekt mit seinem Hut zu fangen, während der andere, vertieft in ein Fossil und die Lektüre im Buch der Natur, im Gras liegt. Dem kleinen Format der Vignette zum Trotz eröffnet sich hier ein ganzer Reigen an Assoziationen und Anspielungen. Zunächst fällt das Sammeln, das Beobachten und Reflektieren als naturkundliche Praxis auf (vgl. Gamper 2013). Hier ist es freilich auch in Bezug auf ästhetische Phänomene wie Gesichterprofile oder Gebährden zu sehen. So hatte kurz vor Erscheinen der Physiognomischen Fragmente Lavaters

\footnotetext{
${ }^{6}$ Der Stamm des Exemplars befindet sich heute noch im Botanischen Garten in Berlin-Dahlem. Vgl. dazu: Sukopp 2011. Zur vielfältigen poetischen Verarbeitung des Experiments vgl. Kittelmann 2018a, S. $60-64$.
} 


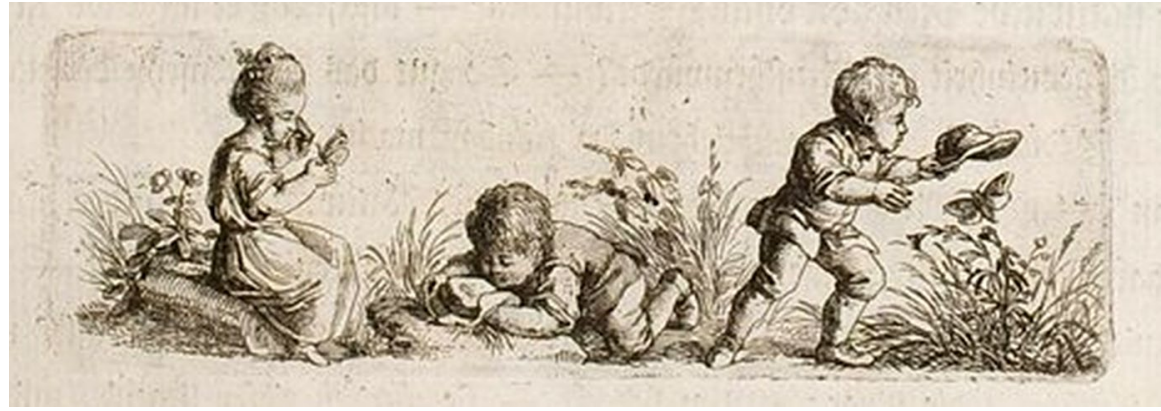

Abb. 4 Vignette, in: Johann Caspar Lavater: Physiognomische Fragmente, Bd. 2, Leipzig 1776, S. 173

Mentor Johann Georg Sulzer in seiner Allgemeinen Theorie der Schönen Künste unter dem Lemma „Gebehrden“ vorgeschlagen:

Wenn man bedenkt, daß mancher Liebhaber der Naturgeschichte vermittelst der Beobachtung, der Zeichnungen und der Beschreibungen, die Gestalt und die Bildung vieler tausend Pflanzen und Insekte, so genau in die Einbildungskraft gefaßt hat, daß er die kleinesten Abaendrungen richtig bemerket; so laeßt sich auch gewiß vermuthen, daß eine, mit eben so viel Fleis gemachte und in Classen gebrachte Sammlung von Gesichtsbildungen und Gebehrden, und also ein daher entstehender eigener Theil der Kunst, eine ganz moegliche Sach sey. Warum sollte eine Sammlung redender Gebehrden weniger moeglich und weniger nuetzlich seyn, als eine Sammlung von abgezeichneten Muscheln, Pflanzen und Insekten? Und warum sollte man, wenn dieses Studium einmal mit Ernst getrieben wuerde, die dazu gehoerige Kunstsprach und Terminologie nicht eben so gut finden koennen, als sie fuer die Naturgeschichte gefunden worden? ${ }^{7}$

Lavaters Physiognomische Fragmente lassen sich geradezu als Einlösung dieser Idee lesen. Zudem wird der Einsatz technischer Instrumente bei der Beobachtung und Erkundung der Natur thematisiert. Mikroskope und Lupen gehören zur Begegnung mit der Natur unweigerlich dazu. Naturkundliche Instrumente, in zeitgenössischen Texten häufiger als Werkzeuge und Hülfsmittel umschrieben, stellen Hilfsmittel für die Sinne dar. Als solche wirken sie einem mangelnden Vermögen der Sinnlichkeit entgegen. Sie werden als helfende, ergänzende, steigernde oder gar neue Sinne dargestellt (Hoffmann 2006, S. 45). So findet sich bereits 1738 in Friedrich Christian Lessers Einleitung $\mathrm{zu}$ seiner Insecto-Theologia folgender Gedanke: „Nachdem aber die Vergrösserungs-Gläser, als das dritte Auge, erfunden worden, hat man noch vieles an den Insecten wahrgenommen, welches ohne deren Hülffe unentdecket geblieben seyn würde, daß man wohl hier mit Recht sagen kan: drey Augen sehen mehr als zwey.“ (Lesser 1738, S. 19) Die Entomologie oder Insektenkunde kann als eines der

7 Sulzer (1771), S. 428. Vgl. dazu auch Décultot (2011), S. 211. 
relevantesten und populärsten Gebiete der Naturkunde in der ersten Hälfte des 18. Jahrhunderts gelten. Zur selben Zeit wie Lessers Insecto-Theologia (1735-1740) erscheinen die weit rezipierten Schriften des bereits 1680 verstorbenen Swammerdam sowie von de Réaumur. In kürzester Zeit gelangen die Namen dieser Forscher und der von ihnen geprägten jüngeren Generation mit Pierre Lyonnet, Abraham Trembley oder Charles Bonnet zu großer Berühmtheit auch auf deutschem Boden. Diese Erfolgsgeschichte-die Entdeckung des Polyps und ihre Beschreibung durch Trembley 1744 ergriff innerhalb eines Jahres ganz Europa-verdanken die genannten Mikroskopisten ihrem Instrument. Neben Antoni van Leeuwenhoek und Jean-Antoine Nollet sind auch Swammerdam und Réaumur ebenfalls als Mikroskopenentwickler und -bauer bekannt. In einer Zeit, in der die Perfektionierung der Instrumente so weit fortgeschritten ist, dass deren optimale Benutzung und die Beobachtungsrichtlinien in eigenen Abhandlungen verhandelt werden, ist es nicht verwunderlich, dass sich ein Diskurs um das tatsächliche Verdienst des Beobachters entwickelt (Hoffmann 2006, S. 30-43). Anleitungen zum richtigen Gebrauch der Linseninstrumente gehören schon in den 1740er Jahren in mehrere Naturlehrbücher, erreichen in den 1770er Jahren einen Höhepunkt und wirken auch auf poetologische Tendenzen der Zeit ein (vgl. Michler 2013; Blome 2017).

Nicht nur die wissenschaftliche Praxis, sondern überhaupt der Vermögensapparat des Menschen stehen dabei in einer Art Erklärungsnot. Neben sekundären Werkzeuge sind die primären, natürlichen Werkzeuge der Sinne gefragt. Eine direkte Linie wird von der Theorie der Empfindungen zur Wissenschaftstheorie gezogen. Wie Hoffmann beispielhaft für diese Zeit an Patrice d'Arcys Abhandlung über die Dauer der Empfindung des Sehens herausstellt, wird die in Frage stehende Sinnlichkeit als ,eine neue Möglichkeit des Wissens“" ${ }^{\text {8 }}$ zugleich gewürdigt und problematisiert. Diese empfindungsästhetische Problemstelle wirkt sich nun durchaus produktiv auf literarische Freiräume wie die Versdichtung oder die Satire aus. Als prominentes Beispiel dafür kann Kästners Ode Betrachtung bey Gelegenheit des Kometen 1742 dienen, die wohl als satirisches Gegenstück zu seinem in Hallers Stil verfassten Philosophischen Gedichte von den Kometen entstanden ist. Zusätzlich zur Liebes- und Geselligkeitssemantik sowie zum witzigen Umgang mit dem eigenen wissenschaftlichen Selbstbewusstsein, die das Gedicht auszeichnen ${ }^{9}$, durchzieht es eine satirische Darstellung des laienhaften, ungeübten Amateurs. Durch Wendungen wie etwa „mit verschiednem Glücke“ oder „das Rohr, das wankt“, wird die mangelndeGeschicklichkeitderkleinen Gesellschaftkritischbeleuchtet:Einernsthafter Gegenstand der Weltweisheit—die Kometenforschung ist zu dieser Zeit noch in vollem Gange_-sinkt zu einem Paradespiel für ,die Schöne“ und erreicht damit die unphilosophischen oder gar dummen Köpfe (Kästner 1773, S. 186f.):

Wohl! endlich hab ich ihn gefunden,

So ruft der dritte halb entzückt;

Er ruft, und sieht sein Glück verschwunden,

\footnotetext{
${ }^{8}$ Hoffmann: Unter Beobachtung, S. 101-114, hier S. 103.

9 Stadler 2003 bietet eine entsprechende Analyse von Kästners Ode (S. 112-119).
} 
Indem die Hand das Rohr verrückt.

Der Schönen Wünschen und Bemühen

Hält selbst den Unbestand nicht fest;

Sie sehn ihn durch die Gläser fliehen,

Wie er das Rohr, das wankt, verläßt.

Was macht den Stern vor uns verschwinden?

Aefft unsern Fleiß wohl sein Betrug?

Nein, ihn gewiß durchs Rohr zu finden,

Sind wir nur nicht geschickt genug.

Geschichte, du sollst mir ietzt zeigen,

Was wir in keiner Fabel sehn.

Heiß Männer von dem Laster schweigen.

Mit welchem sie die Schönen schmähn.

Man braucht nicht lange nachzusinnen,

Wenn mancher sie für falsch erklärt;

Ihr Herz zu kennen, zu gewinnen,

Fehlt ihm Geschicklichkeit und Werth.

Die Benutzung des Fernrohrs beschränkt sich nicht nur auf eine gelehrte Praxis, sondern wird am Ende des Gedichts auf das männliche Brautwerben übertragen. Der letzte Vers schließt mit einer Moralsentenz, die zugleich die lebensweltliche Relevanz der Naturkunde pointiert andeutet. Instrumente verlangen Geschicklichkeit und werden als Objekte auch mit Fleiß studiert. Hierbei erscheint der individuelle Umgang und die persönliche Auseinandersetzung mit den Apparaten zentral, wie der häufig auch in Briefen kultivierte Austausch über Mikroskope, Barometer und andere Geräte belegt. ${ }^{10}$ Selbst Dichter wie Johann Jakob Bodmer, die an diesen physikalisch-technischen Diskursen kaum teilnahmen, profitierten von diesem Austausch und reflektierten die Entwicklung in ihren Werken. In dem Bibelepos Der Noah bilden Formen und Praktiken der Naturerfahrung und -erkenntnis mit Hilfe technischer Instrumente wiederholt den Hintergrund der vorzeitlichen bzw. vorsündflutlichen Thematik. So trägt die Figur des Sipha Züge eines Naturforschers der Aufklärung, der für seine Töchter einen botanischen Garten anlegt und dem sich das Buch der Natur ,,vor seinen Augen eröfnet, [a]ls ihm gegeben ward, Krystall in Linsen zu schleifen“. Sipha gibt seine Kenntnisse an seine Verwandten weiter. Er lehrt sie ,die Wunder der Linse“ und blickt mit einem Teleskop auf „,neue Planeten“, auf die ,Thäler und Berg in dem Monde der unsern Erdball begleitet“. ${ }^{\text {" }}$

\footnotetext{
${ }_{10} \mathrm{Vgl}$. die in großen Teilen noch unpublizierten Briefwechsel von Johann Georg Sulzer, Johann Heinrich Lambert, Johannes Gessner oder Georges-Louis Le Sage.

11 Bodmer 1752, S. 175f. Informationen über naturhistorische und technische Entwicklungen erhielt Bodmer vor allem über die Briefe seines Schülers und Freundes Johann Georg Sulzer. Der Briefwechsel wird 2020 im Schwabe-Verlag und kurze Zeit später auch als Online-Edition erscheinen.
} 


\section{Natur im Brennglas}

Bodmers Schüler Johann Caspar Lavater arbeitete als junger Mann ebenfalls an einer „Verbeßerung der Perspektive“ (Bodemann 1878, S. 208) und baute an einem Teleskop und einem Mikroskop, die er kostengünstig unter die Leute bringen wollte. In derselben Zeit begann er, angeregt durch seinen Freund, den Mediziner Johann Georg Zimmermann, sich physiognomischen Studien zu widmen. Auch Insekten spielen in seinen Fragmenten eine nicht unbedeutende Rolle. Auf zahlreichen Tafeln werden z. B. detaillierte Abbildungen von Käfern, Schmetterlingen und Raupen dem Publikum präsentiert. Dabei scheint die konkrete Beobachtung, Durchdringung und Verdinglichung der Natur, die Lavater hier propagiert und weiterentwickelt, durchaus noch unter dem Einfluss physikotheologisch geprägter Naturforscher und Literaten wie Barthold Heinrich Brockes und Albrecht von Haller zu stehen, die schon in den ersten Jahrzehnten des 18. Jahrhunderts die Benennung, (mikroskopische) Betrachtung und Beschreibung von naturkundlichen Objekten (vor allem Pflanzen) zum Kern ihres poetischen Schaffens erhoben (Richter 1972, S. 199-202). In Hallers Die Alpen (1729) sammeln sich deren Bewohner und Hirten um einen Naturforscher, der die Züge des Naturforschers und Alpinisten Jakob Scheuchzer trägt, und als Sammler und Klassifikator einheimischer Kräuter und Moose bewundert wird:

Bald aber schließt ein Kreis um einen muntern Alten,

Der die Natur erforscht und ihre Schönheit kennt;

Der Kräuter Wunder-Kraft und ändernde Gestalten

Hat längst sein Witz durchsucht und jedes Moos benennt;

Er wirft den scharfen Blick in unterirdsche Grüfte,

Die Erde deckt vor ihm umsonst ihr falbes Gold,

Er dringet durch die Luft und sieht die Schwefel-Düfte,

In deren feuchter Schoß gefangner Donner rollt;

Er kennt sein Vaterland und weiß an dessen Schätzen

Sein immerforschend Aug am Nutzen zu ergötzen. ${ }^{12}$

Haller ging es nicht zuletzt darum, einen wissenschaftlich-gelehrsamen und zugleich sinnlichen Zugang zur Natur in seine Dichtung zu integrieren, was ihm unter anderem den Spott der anakreontischen Dichter einbrachte. So dichtete Gleim (1753, S. 29):

Herr Haller sucht Gras, Kraut und Bäume, auf mancher rauhen Bahn;

Ich Klügerer, ich suche Reime,

So wie er sonsten auch gethan.

Dennoch kommt der Botanik als Wissenschaft des Sichtbaren auch innerhalb dichterischer Prozesse, Stoffe und Motive eine exponierte Stellung zu. Im Suchen und Besuchen, Sammeln und Erhalten von Pflanzen artikulieren sich zugleich die

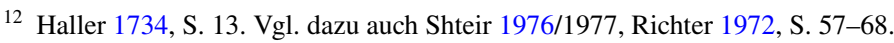


Suche nach Gott und die Offenbarung göttlicher Größe. Akribisch, detailreich und ,vor Verwund'rung starr“ geschildert, zeigt sich etwa bei Barthold Heinrich Brockes in Hyazinthen, Vergissmeinnicht, Tulpen oder schlichten, winzigen Samen=Gehäusen Gott in der Natur. Begünstigt durch den Fortschritt im Bau von Mikroskopen und Teleskopen vermischen sich in physikotheologischen Texten Mikro- und Makrokosmos, Natur-, Wahrnehmungs- und Moralbetrachtungen miteinander. So schreibt Lavaters Freund und Mentor Johann Georg Sulzer, der auch mit Haller eine ausführliche Korrespondenz pflegte, in seinem 1745 publizierten Versuch einiger Moralischen Betrachtungen über die Werke der Natur (Sulzer 1745, S. 2):

Die kleinen Körperchen, welche Löwenhuk durch die Vergrösserungsgläser entdecket, und die weit kleinere, welche Leibniz durch Vernunftschlüsse gefunden hat, sind eine reiche Quelle des Wunderbaren, insonderheit vor diejenigen, welche ihr schwaches Auge zum Richter über das Grosse und Kleine in körperlichen Dingen gesetzt haben.

Einflüsse davon sind noch bei dem jungen Christoph Martin Wieland in seinem philosophischen Lehrgedicht Die Natur der Dinge zu finden, obgleich sich darin bereits ein etwas verändertes Verhältnis von instrumenteller Erkenntnis und rationaler Spekulation andeutet. Maßstab ist bei Wieland nunmehr die Vernunft als Vermögen ins Unerkennbare einzudringen. Die mit technischen Apparaten versehene Wissenschaft besitzt die Rolle einer bestätigenden, nachrangigen Instanz (Wieland 1752, S. 89):

So gründet unsern Satz, den die Vernunft gebeut

Auch der Erfahrung Spruch, und hilft der Sinnlichkeit.

Doch kein vergrößend Glas führt die geschärften Blicke

Aufs unterste Geschlecht der Creatur zurücke.

Denn diese kleidt ein Leib vom feinsten Stoff erbaut,

Den selbst kein Leeuwenhök, kein Needham nicht beschaut.

Zugleich war Wieland fasziniert von Pflanzen, deren Morphologie er wie unter einem Mikroskop literarisch fixiert und die er aller Kunst, ja sogar der populärsten Skulptur der Neuzeit, dem unter anderem von Lessing und Winckelmann beschriebenem Laokoon vorzieht (ebd. S. 17):

Und was ist jenes Werk [Laokoon], das aller Griechen Blick

Mit Rührung auf sich zog, des Meißels Meisterstück,

Nur gegen einen Staub, aus dem die Pflanzen sprossen,

Wo unbegreiflich klein, von mancher Haut umschlossen,

Die künft'ge Blume liegt, geformt doch unbelebt,

Aus tausend Fäserchen mit weiser Kunst gewebt?

Unendlich ist für uns der zarten Fibern Länge,

Unzählbar unserm Blick der kleinen Adern Menge,

Die nach dem Grundgesetz, das in den Wesen liegt,

Die wirksame Natur unendlich schön gefügt. 


\section{Zu den Dingen oder der Text als Vitrine}

Doch die mikroskopische Betrachtung allein genügt nicht. Um Erkenntnis zu erlangen, muss man unweigerlich das Gelehrtenzimmer verlassen und die Begegnung mit Pflanzen oder Gesteinen, die sich selbst nicht fortbewegen können, suchen. Das wissen auch die drei Figuren in Lavaters Fragmenten. Naturkundliches, insbesondere botanisches und geologisches Beobachtungsprimat und poetisch wahrnehmbare und darstellbare Schönheit greifen ineinander. Beobachten, Sammeln, Ordnen, Empfinden und dichterische Vergegenwärtigung (bzw. Lektüre) von Pflanzen, Insekten und anderen naturkundlichen Objekten vereinen sich zu einem festen Erkenntnis- und Schreibmodell. Dazu gehört, dass man zu den Dingen gehen, sie in bestimmten Räumen aufsuchen muss, bei einem Spaziergang, einer Wanderung, einer Reise oder einer Exkursion. Haller und Sulzer (später auch Lavater) ${ }^{13}$ misstrauen ,einer Überbewertung der Verstandeskräfte" und setzen auf die bereits von ihrem Lehrer Johann Jakob Scheuchzer etablierte sinnliche Erfahrung von Natur. Sie plädieren dafür, bekannte Wege zu verlassen und sich buchstäblich in unwegsames Terrain, ,aus dem Cabinette heraus mit dem Auge des Forschers“ zu begeben und „Gründe und Thäler, die dunckele und unwegsame Wälder [zu] durchsuchen.“ (Sulzer 1747, S. 9)

Das Gehen zu den Dingen, das Aufsuchen und Durchsuchen der Natur, das Sammeln von Objekten, die Vermessung und Karthographierung der Landschaft gewinnen demnach in Texten der Aufklärung an enormer Bedeutung. Die narrativ-mobile Aneignung der Natur geht mit deren technisch-wissenschaftlicher Erschließung einher. So schildert etwa der von Haller und Sulzer protegierte Christlob Mylius auf seiner zu Ostern 1753 absolvierten Reise auf den Blocksberg (Brocken), wie er extra einen „Führer“ mitnimmt, der ,[s]eine Instrumente trug“. Bei den Instrumenten handelt es sich um Barometer und Thermometer, die immer wieder zum Einsatz kommen. Auf dem verschneiten Gipfel angelangt verzeichnet Mylius die genauen Temperatur- und Luftdruckverhältnisse (Mylius 1753, S. 1027):

Ich machte gleich meine Instrumente mit aller Sorgfalt zurechte, und beobachtete auf einem Steinhaufen auf der äußersten Spitze des Berges Folgendes früh um $6 \mathrm{Uhr}$, bey hellem Wetter und schwachen doch scharfen Südostwinde:

Barometer: 2411 parisische Maaß.

Thermometer: 36 Grad über o Fahrenh.

Neben meteorologischen Phänomenen räumt Mylius naturkundlichen Objekten viel Raum ein. Linnés Klassifizierung und Einteilung der Natur in drei Reiche aus Mineralien, Pflanzen und Tieren (vgl. dazu Lepenies 1988, S. 9-61) folgend vermerkt er z. B. „Violensteine“ (Mylius 1753, S. 1024), „schöne bunte Mooße“,

\footnotetext{
${ }^{13}$ Bei Lavater emanzipiert sich der Diskurs über Beobachtung als eine gelernte Fähigkeit und schließt sich bald dem Geniegedanken an. Quasi zeitgleich wie in Jean Senebiers bekannter Schrift über die Kunst der Beobachtung von 1775 stellt man in Lavaters Physiognomik ebenfalls eine Verschiebung von der reinen Praxis und Übung der Beobachtung hin zur Behauptung einer Beobachtungsgabe fest. Diese macht sich in Form des Genies bemerkbar und beansprucht eine über die Wahrnehmung der Natur hinausgehende Ahnung des Geistes. Vgl. den Abschnitt „Die Physiognomik, eine Wissenschaft“ in Lavater 1775 , S. 52-56.
} 
„Erica und Myrtillus“ (ebd. S. 1028) und findet in den Torfhäusern am Fuße des Brockens „einen Papilion von der gemeinen gelb und schwarzen Dornenraupe“ (ebd.). Die Struktur bzw. die Ordnung des Textes scheinen damit der Ordnung eines Naturalienkabinetts zu folgen. Der Brocken wird zum naturkundlichen Wissensraum und der über den Berg verfasste Text zu einer Art Schauraum, einer virtuell-narrativen Vitrine (vgl. Benjamin 1966, S. 174, sowie Dolezel 2017), durch die der Leser auf den Brocken und dessen naturkundliches Interieur sehen kann. Die von Walter Benjamin diagnostizierte „tiefste Bezauberung des Sammlers“ (Benjamin 1966, S. 174), die sich bei aller rationalen Nüchternheit in den Bemerkungen von Mylius über die karge Flora und Fauna des Brockens dennoch widerspiegelt, wird so über den Text auf den Leser übertragen.

Moose hatten es auch der wie Mylius in Berlin lebenden Dichterin Anna Louisa Karsch angetan. Das Harz-Moos, eines ihrer einprägsamsten Gedichte, trägt den Untertitel Als Herr Dohmdechant Freyherr Spiegel zum Diesenberg etwas Moos vom Harzgebürge mitgebracht hatte. (Zu Halberstadt den 10ten des Weinmonaths 1761) und verweist auf die Beobachtung, Sammlung, Weitergabe und anschließende lyrische Transposition von Pflanzen. Anders als bei Mylius, der stets die naturkundliche rationale Distanz zu den Dingen wahrt und sich auf die Benennung und Charakterisierung als ,schön“ beschränkt, mündet die Begegnung mit dem Moos hier in einer Selbstreflexion der Dichterin, die ursprünglich Viehhirtin in Schlesien war, in Berlin als Naturgenie gefeiert und durch ihre Stegreifpoesie schnell zur Berühmtheit wurde und sich in dem dauerhaften und widerständigen Moos selbst erkannte (Karsch 1764, S. 339f.):

Wie ähnlich ist es mir! tief lag ich unter Gram

Viel schwere Jahre lang, und als mein Winter kam,

Da stand ich unverwelkt und fieng erst an zu grünen.

Ich muste, wie das Moos, dem Glück zum weichen Tritt,

Dem Thoren zur Verachtung dienen.

Einst sterb ich! Doch mein Lied geht nicht zum Grabe mit!

Die Metaphorisierung des Mooses steht zugleich für eine vitale Schöpfungspoetik der Dichterin, die sich als gewachsen beschreibt und auch von Zeitgenossen immer wieder so beschrieben wurde (vgl. Mödersheim 1995). Johann Peter Uz und Moritz von Thümmel etwa attestierten der Karschin, dass sie ihr Talent in „,bemoosten Gründe[n]“ wo sie ihre Rinder weidete, ,ungesucht“" gefunden habe. ${ }^{14}$ Mylius' Text erreicht dabei nicht die poetische und reflexive Dimension des Gedichtes der Karschin über das ihr mitgebrachte „Harz-Moos“, beruht im Grunde aber auf identischen Praktiken des Sammelns und der damit verbundenen (textuellen bzw. haptischen) Weitergabe naturkundlichen Wissens.

Naturkundliches Interieur, Apparaturen und Objekte bzw. das Wissen darüber waren ab 1750 breit verfügbar und erlangten sowohl als Gegenstand als auch als Darstellungsweise, die einen nicht alltäglichen Wahrnehmungsprozess reflektieren, den Rang eines literarischen und publizistischen Motivs. In hauptsächlich privat

${ }^{14}$ Moritz von Thümmel: Auf die Frau Karschin. Hier zit. n. Schüddekopf 1899, S. 281. 


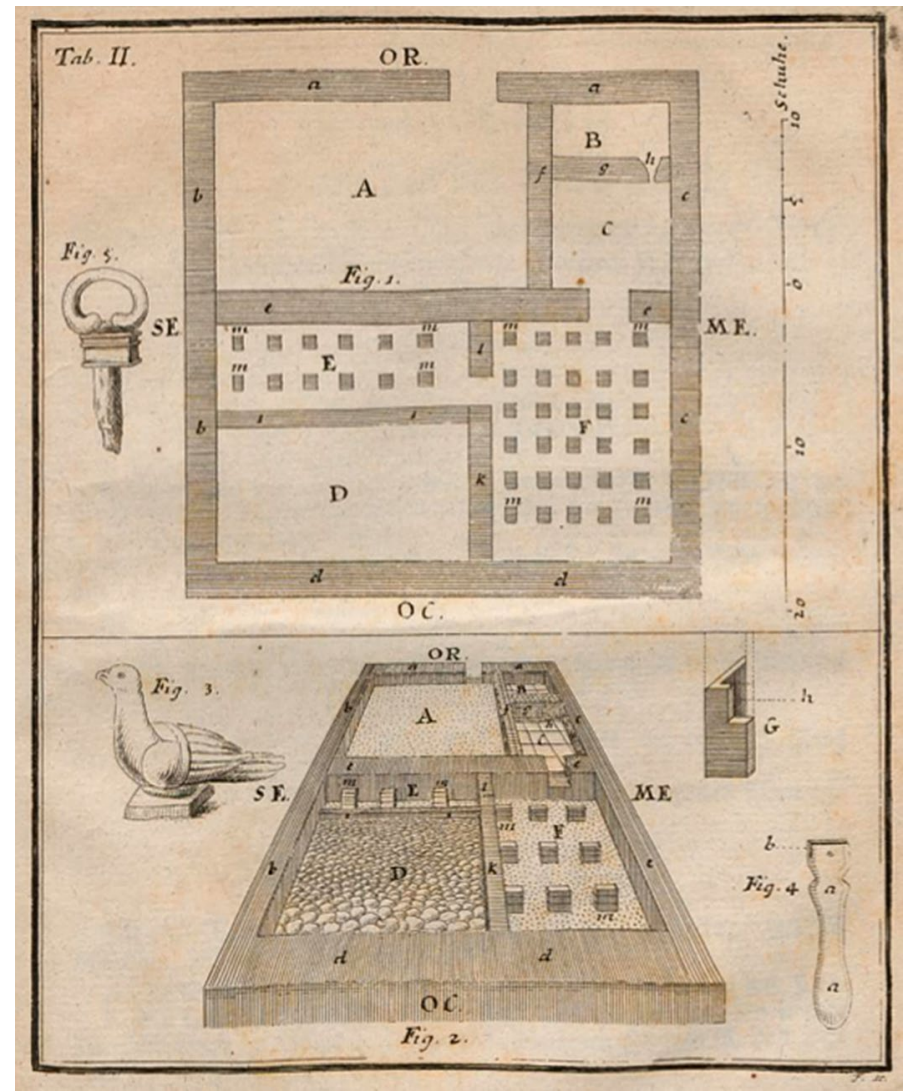

Abb. 5 Tafel in: Johann Georg Sulzer: Ausführliche Beschreibung einer Merkwürdigen Entdeckung, verschiedener Antiquitäten: In dem, in der Herrschafft Knonau gelegenen Dorff Nider-Lunneren, in dem Jahr 1741. Zürich 1742, Anhang (unpag.)

gehaltenen Forschungs- oder Betrachtungsstunden war es dem Liebhaber der Natur allmählich gegeben, eigene, zum Teil nirgendwo anders dokumentierte Erkenntnisse zu gewinnen und sie einem breiteren Publikum in unterschiedlicher textueller Form darzulegen. Davon zeugen auch die Beiträge des sich stets an der Schnittstelle zwischen Naturforschung, Ästhetik und Dichtung bewegenden Johann Georg Sulzers. Der aus Winterthur stammende Sulzer, seit 1750 Mitglied der Akademie der Wissenschaften und eine Art Knotenpunkt der Berliner Aufklärung, war gern in realen Räumen, in Bergen, auf Feldern, auf Chausseen oder in Gärten unterwegs und sammelte Wissen, das er sowohl an befreundete Gelehrte (vgl. dazu Kittelmann 2018b) als auch ein breites Publikum weitergab. Einige Jahre vor Mylius reiste er auf den Brocken und ließ in seine Text- und Reisepraxis Kenntnisse einfließen, die er vorab schon auf umfangreichen Exkursionen in den Alpen und im Alpenvorland erfolgreich angewandt und an die „Liebhaber der Naturhistorie“ (d. h. seine Leser) vermittelt hatte. Unter den beobachteten, entdeckten oder gesammelten Objekten, die 


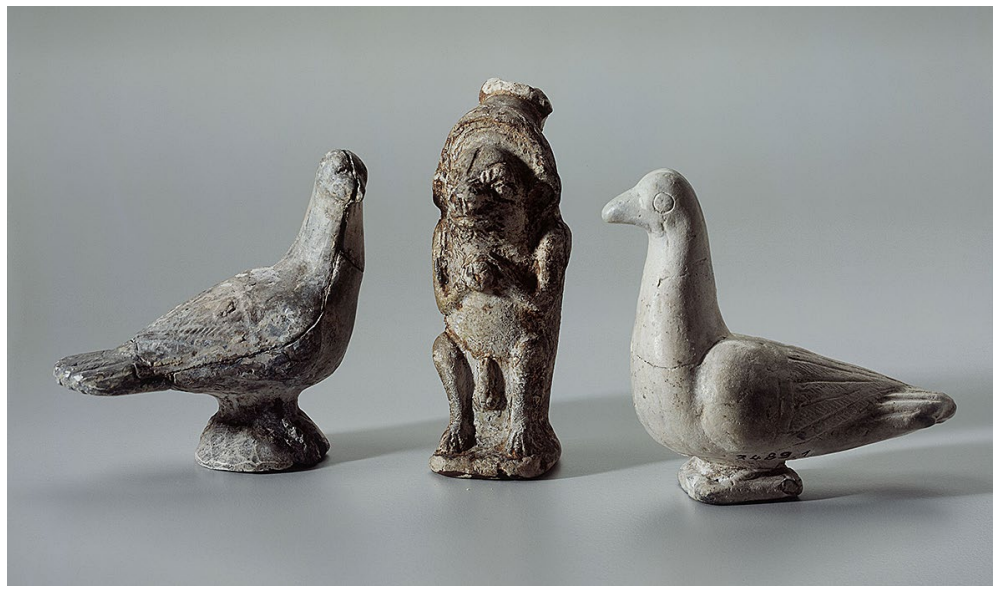

Abb. 6 Von Johann Georg Sulzer in Lunnern gefundene archäologische Objekte, Schweizerisches Landesmuseum Zürich

Sulzers Beschreibungen detailliert präsentieren, sind u.a. „sehr schöne Petrefakte“, Ammoniten, Pflanzen, aber auch antiquarische Funde wie kleine Tier- und Gottheitenstatuen, die Sulzer in dem kleinen Schweizer Dorf Lunnern (Abb. 5 und 6) bei archäologischen Grabungen entdeckt und als Erster beschrieben hatte. Wie ein von ihm selbst entwickeltes Reisethermometer, das ihn überall begleitete (noch auf seiner Altersreise nach Südfrankreich 1775 wird er es mit sich führen), bildete Sulzer diese Funde zuweilen auf dem Text beigefügten Tafeln ab. Die Dimension des Textes wird so noch erweitert. Zur „Erzehlung“ naturkundlicher Dinge und Artefakte gesellt sich die Ebene der konkreten Anschauung. Die Topographie und textinterne Repräsentation der Reise wird durch Kartenmaterial, Tafeln und Tabellen veranschaulicht und erweitert. Die Publikation bzw. das Buch avanciert zu einem materialreichen, vielfältigen und mediale Formen übergreifenden Wissensraum (vgl. Wolf 2015), zu einem Ort der Wissensproduktion, der zwischen materiellen (Schrift, Bild, Objekt) und darstellungslogischen (Tafeln, Tabellen, Karten) Ebenen oszilliert. Sulzer reagierte damit auch auf die Erwartungen eines Publikums, das naturkundliche Dinge und Objekte in erster Linie sehen und weniger darüber lesen wollte. Sehen und Beobachten übten direkten Einfluss auf das Erscheinungsbild von Büchern aus. In seiner Vorrede zum zweiten Teil der von ihm herausgegebenen Natur-Geschichte des Schweitzerlandes Johann Jakob Scheuchzers bemerkte er: „Viele Leute lieben die Kupferstiche weit mehr, als eine Beschreibung der Sachen; ja einige kauffen die Bücher nur deßwegen.“ (Sulzer 1746, S. VI) 


\section{Die Schönheit des Naturalienkabinetts oder die Herrschaft über die Dinge}

Sulzer betrieb jedoch nicht nur klassifizierende Naturgeschichte, sondern übertrug seine naturkundlichen Forschungen und Erkenntnisse in ästhetische, philosophische und poetische Kontexte. Insbesondere wird das in seinen 1750 publizierten Unterredungen über die Schönheit der Natur deutlich. Die an Shaftesburys Moralists orientierten und als hymnische Dialoge zwischen den beiden Figuren Eukrates und Charites ${ }^{15}$ konzipierten Gespräche kreisen unter anderem um die Schönheit, Proportion und den harmonischen Bau der Pflanzen, um die Seltenheiten und Wunderbares im Reich der Mineralien, um die Verwandlung der Insekten oder die Haushaltung und das Genie der Bienen. In den Unterredungen vermischen sich die botanische, zoologische und geologische Fachkenntnis ihres Verfassers mit dem Anspruch, die Natur als Schöpfung Gottes und damit als Raum moralischer Erbauung zu preisen. Die Natur und ihre Dinge und Objekte werden als „unermeßliche[s] Feld der Schönheit““ (Sulzer 1750, S. VI) gepriesen. Sulzer geht es darum, den ,innerlichen Werth der Materie zu erkennen“ (ebd. S. XIII) und zugleich Wissen über die Dinge daraus abzuleiten. In der Vorrede steht diesbezüglich zu lesen (ebd. S. XVI):

Wir machen es nicht besser, wenn wir von dieser Erde ziehen, ohne die Natur gekannt zu haben. Alles ist ohne Zweifel zu unserm Vortheil darin angelegt. Was für ein herrliches Leben könnten wir führen, wenn wir den rechten Gebrauch eines jeden Dinges wüßten, wenn wir die Natur kennten! Ich glaube denen einen wichtigen Dienst zu thun, die ich zur genauen Betrachtung der Natur ermuntern kan.

Die erkennende Betrachtung der Natur läuft gleichsam in Sulzers Unterredungen in unterschiedlicher Weise ab: als Spaziergang durch die freie Flur und das Feld oder den Wald, wo Dinge wie „Steine und Wurzeln der Eichen und Tannen-Bäume“ oder ein „Schaz von Blumen“ (ebd. S. 19) die Aufmerksamkeit wecken. Oder im Garten, wo ,wolriechende Gewürze“ die Sinne erregen und Samengehäuse zum „Gebrauch des Vergrösserungs Glases“ einladen: „Dies sind andre Augen, die uns mit einer neuen Welt von Wundern bekannt machen." (ebd. S. 48) Mit der wiederum thematisierten mikroskopischen Beschreibung der Pflanze ist sowohl Erkenntnis als auch eine Art ästhetische Lust verbunden, die dem Text inhärent ist. Pflanzen haben einen direkten Zugang zum Herzen und Empfindungsleben der Menschen und stehen zugleich für einen zentralen aufklärerischen Verwandlungstopos: die Wahrnehmung von Pflanzen wird in Gefühl und/oder moralische und wissenschaftliche Erkenntnis umgewandelt. In diesem Sinne legt Sulzer seinem Eukrates die Worte in den Mund: „eine einzige Pflanze [...] wird dich mehr Erfindung sehen lassen, als alles was dir iezo bekannt ist." (ebd. S. 40)

Der Spaziergang durch verschiedene Erkenntnis- und Anschauungsräume endet schließlich im Naturalienkabinett, dessen Schönheit ein eigener Abschnitt gewidmet

${ }^{15}$ Eukrates trägt die Züge von Sulzers Lehrer, den Botaniker Johannes Gessner, und Charites erinnert an Sulzer selbst. 
ist. Hier wird die gesamte Natur im wahrsten Sinne visuell erfahrbar und dinglich veranschaulicht: „Denn da habe ich, so viel möglich ist, die Natur ins kleine gesammelt. Da wirst du alles mit Augen sehen können, was ich dir von der Natur gesagt habe“ (ebd. S. 31), heißt es im Dialog zwischen Eukrates und Charites. Vorbild ist in diesem Falle das Zürcher Naturalienkabinett von Johannes Gessner, ${ }^{16}$ wo sich Sulzer als junger Mann tatsächlich für seine Naturstudien aufhalten durfte und dessen mehr als tausend Pflanzen umfassende Herbarien er benutzte. Als zentrale Konfiguration des 18. Jahrhunderts ist das Kabinett sowohl Wissensraum als auch Quelle und Ort ästhetischer Inspiration. So vermerkt Eukrates, dass man die Harmonie der „schönst[en] Statüe“ nicht ohne „Kenntniß der Natur“ empfinden könne und dass „die Natur die Ursprüngliche Werkstäte aller Künste“, eine ,unendliche Rüstkammer der künstlichsten Maschinen[sei], die alles, was die Menschen erfunden haben, weit übertreffen.“ (ebd. S. 39) Zugleich geht es um eine Herrschaft über die Dinge, die man sich hier aus der Welt in das Kabinett, aus dem Außenraum in den Innenraum und schließlich auch in den Text holt. Anders als in der freien Natur, die durch den Wandel der Jahreszeiten geprägt ist, ,wo der Winter einen Schleyer über die Schäze der Natur ziehet" (ebd. S. 32), stehen naturkundliche Dinge im Kabinett dem Betrachter immer zur Verfügung: „Er ist immer Herr davon, es stehet immer in seiner Gewalt“, betont Sulzers Eukrates. Indem die Dinge dem häuslichen Interieur, einverleibt" werden, unterliegen sie permanent dem Blick und dem Zugriff des Besitzers, der sie ordnen, klassifizieren, bewerten und nahezu panoramatisch übersehen kann: ,Das Reich der Pflanzen bleibt mir in meinen Zimmern immer grün, und was man hier und da zerstreuet findet, habe ich vereiniget um die Natur mit einem Blikke zu übersehen." (ebd. S. 33) Diese mit dem Naturalienkabinett verbundene spezifische Sehweise auf naturkundliche, gesammelte (und musealisierte) Dinge tangiert letztendlich auch die Verdinglichung von Sulzers Text, der ebenfalls einzelne Objekte in den Fokus seiner Unterredungen über die Natur und deren vielfältige Schönheit und Erscheinungsformen rückt. Ähnlich wie im Kabinett wird damit im Text durch die poetische Aneignung eine verstetigte Präsenz naturkundlicher Dinge erreicht und popularisiert.

\section{Epilog}

Für Naturforscher wie Albrecht von Haller oder Johann Georg Zimmermann verbanden sich mit dieser optimistischen Haltung zu den Dingen, Interieurs und Werkzeug freilich auch Schwierigkeiten. Haller und sein Schüler Zimmermann hatten durchaus Vorbehalte sowohl der positiven Physikotheologie als auch der Popularisierung der Naturforschung und deren technischen Möglichkeiten und breitenwirksamen dinglichen Vergegenwärtigung gegenüber. Bereits in den frühen Gedichten Albrecht von Hallers deutet sich ein pessimistisches Bild vom Menschen (,ein Mittel-Ding von Engel und von Vieh“) an, das die Fortschritte in der

\footnotetext{
16 Vgl. zu Gessner und Sulzer: Boschung 1996, S. 17ff. Gessner stand unter anderem in regem brieflichbotanischen Austausch mit Albrecht von Haller. In dem Briefwechsel wird Sulzer mehrfach erwähnt.
} 
Wissenschaft, die inflationäre Sammlung und Präsenz naturkundlicher Dinge und insbesondere die Instrumente als nutzlose Werkzeuge in den Händen der Menschen präsentiert:

Der Weise braucht umsonst, geführt von der Natur

Das Bleymaaß in der Hand, und die Vernunft zur Schnur.

Im weiten Labyrinth wahrscheinlicher Begriffen

Kan auch der Klügste sich in fremde Bahn vertieffen,

Und wann sein sichrer Schritt sich nie vom Pfad vergißt,

So sieht er doch am End, daß er am Anfang ist. ${ }^{17}$

Zimmermann, in späteren Jahren ein enger Freund Sulzers, stand in derselben Tradition und tendierte in eine ähnliche Richtung. In seinen Betrachtungen über die Einsamkeit beschäftigt er sich mit Naturwissenschaftlern, die ,ihr Leben mit Betrachtung der Insecten zubringen“ und doch „,nichts neues“ sehen. Mit einem Zitat von Diderot bemerkt er: „Grossen Geistern gehören grosse Objecte, kleine Objecte sind für kleine Geister geschaffen“ und warnt zugleich davor ,die Absichten der Natur erklären [zu] wollen“ oder gar „Gott in den Falten der Haut des Rhinoceros zu finden“ (Zimmermann 1756, S. 42).

Hallers und Zimmermanns Skepsis verweist dabei auf unterschiedliche Formen der Annäherungen an naturkundliche Diskurse und deren Verarbeitung in literarischen Texten der Aufklärung. Das trifft insbesondere auch für die Präsenz naturkundlicher Interieurs und Objekte zu. So verschieden die Gattungen sind, in denen sie zu finden sind (z.B. in Lehrgedichten, in der Erlebnislyrik, in Epen, moralphilosophischen Abhandlungen oder in Journalbeiträgen), so verschieden ist auch ihre Rolle innerhalb der Texte: Als Attribut, Metapher, sinnstiftendes Motiv, Reflexionsmedium oder ästhetisches Beobachtungsobjekt werden sie ebenso verhandelt und eingesetzt wie als Hintergrund des poetischen Geschehens. In jedem Falle verweisen sie auf eine für die Epoche der Aufklärung zentrale Konstellation und Schnittstelle zwischen Naturwissenschaft, Dichtung und Ästhetik, die schließlich sowohl die Art der Darstellung von materiellen Objekten als auch die äußere Ausstattung der Texte selbst beeinflusst und auch für spätere Epochen-erinnert sei hier nur an das Naturalienkabinett in Chamissos Peter Schlemihl oder E.T.A. Hoffmanns Linsenschleifer Coppelius_-nichts an Faszination verloren hat.

Acknowledgements Open Access funding provided by Projekt DEAL.

Open Access This article is licensed under a Creative Commons Attribution 4.0 International License, which permits use, sharing, adaptation, distribution and reproduction in any medium or format, as long as you give appropriate credit to the original author(s) and the source, provide a link to the Creative Commons licence, and indicate if changes were made. The images or other third party material in this article are included in the article's Creative Commons licence, unless indicated otherwise in a credit line to the material. If material is not included in the article's Creative Commons licence and your intended use is not permitted by statutory regulation or exceeds the permitted use, you will need to obtain

${ }_{17}$ Albrecht von Haller: Gedanken über Vernunft, Aberglauben und Unglauben an Herrn Professor Stähelin (1729). In Haller 1734, S. 53-68, hier S. 53. 
permission directly from the copyright holder. To view a copy of this licence, visit http://creativeco mmons.org/licenses/by/4.0/.

\section{Literaturverzeichnis}

Baasner, R. (1987). Lob der Sternenkunst. Astronomie in der deutschen Aufklärung. Göttingen: Vandenhoeck und Ruprecht.

Baasner, R. (1991). Abraham Gotthelf Kästner. Aufklärer (1719-1800). Tübingen: Niemeyer.

Benjamin, W. (1966). Ich packe meine Bibliothek aus-Eine Rede über das Sammeln (1931). In Angelus Novus-Ausgewählte Schriften 2 (S. 169-178). Frankfurt am Main: Suhrkamp.

Berndt, F., \& Fulda, D. (Hg.). (2012). Die Sachen der Aufklärung. Beiträge zur DGEJ-Jahrestagung 2010 in Halle an der Saale. Hamburg: Meiner.

Bianchy, J. F. v. (1762). Das Merkwürdigste vom Barometre und Thermometre. In sieben Abschnitte zusammen getragen und mit einer neuerfundenen Wetterglas-Tafel versehen. Wien: Trattner.

Blome, E. (2017). Künstliche Klassen: Zur Naturaliensammlung in der Literatur des 19. Jahrhunderts. In M. Neumann, A.-M. Post, F. Schneider und M. Twellmann (Hg.), Modernisierung und Reserve. Zur Aktualität des 19. Jahrhunderts (S. 43-61). Stuttgart: Metzler.

Bodemann, E. (1878). Johann Georg Zimmermann, sein Leben und bisher ungedruckte Briefe an ihn. Hannover: Hahn.

Bodmer, J. J. (1752). Der Noah in zwölf Gesängen. Zürich: Gessner.

Boschung, U. (1996). Johannes Gessner (1709-1790). Der Gründer der Naturforschenden Gesellschaft in Zürich. Seine Autobiographie: aus seinem Briefwechsel mit Albrecht von Haller. Ein Beitrag zur Geschichte der Naturwissenschaften in Zürich im 18. Jahrhundert. Zürich: Neujahrsblatt der Naturforschenden Gesellschaft.

Dawson, R. (1976). Collecting with cook: The forsters and their artifact sales. The Hawaian Journal of History, 13, 5-16.

Décultot, E. (2011). Sulzers „System der schönen Künste“. In F. Grunert und G. Stiening (Hg.), Johann Georg Sulzer (1720-1779). Aufklärung zwischen Christian Wolff und David Hume (S. 211-226). Berlin: Akademie Verlag.

Décultot, E., Kampa, P., \& Kittelmann, J. (Hg.). (2018). Johann Georg Sulzer: Aufklärung im Umbruch. Berlin und Boston: de Gruyter.

Dolezel, E. (2017). Die Logik des Schauraums. Zur Präsentation von Naturalien abseits der Taxonomien. In S. Förschler und A. Mariss (Hg.), Akteure, Tiere, Dinge. Verfahrensweisen der Naturgeschichte in der Frühen Neuzeit (S. 208-223). Köln, Weimar, Wien: Böhlau.

Forrer, T. (2013). Schauplatz/Landschaft: Orte der Genese von Wissenschaften und Künsten um 1750. Göttingen: Wallstein.

Foucault, M. (2015). Die Ordnung der Dinge. Eine Archäologie der Humanwissenschaften (U. Köppen, Übers.). 25. Aufl. Frankfurt am Main: Suhrkamp.

Gamper, M. (2013). Beobachten. In R. Borgards, H. Neumeyer, N. Pethes und Y. Wübben (Hg.), Literatur und Wissen. Ein interdisziplinäres Handbuch (S. 241-254). Stuttgart und Weimar: Metzler.

Gleim, J. W. L. (1753). Versuch in Scherzhaften Liedern. 2. Aufl. Berlin: o.V.

Haller, A. v. (1734). Versuch von schweizerischen Gedichten. Bern: Haller.

Hochadel, O. (2003). Öffentliche Wissenschaft: Elektrizität in der deutschen Aufklärung. Göttingen: Wallstein.

Hoffmann, C. (2006). Unter Beobachtung. Naturforschung in der Zeit der Sinnesapparate. Göttingen: Wallstein.

Karsch, A. L. (1764). Auserlesene Gedichte. Berlin: Winter.

Kästner, A. G. (1773). Vermischte Schriften. Bd. 1. 2. Aufl. Altenburg: Richter.

Kimmich, D. (2018). Dinge in Texten. In S. Scholz \& U. Vedder (Hg.), Handbuch Literatur und materielle Kultur (S. 21-28). Berlin und Boston: de Gruyter.

Kittelmann, J. (2018a). Apoll und Minerva. Botanisch-ästhetische Konstellationen in der Literatur der Aufklärung. In Dies. (Hg.), Botanik und Ästhetik (S. 57-78). Göttingen: Universitätsverlag.

Kittelmann, J. (2018b). „Sylvan und die Dryaden gehen noch über die Musen.“ Botanisches Wissen in Johann Georg Sulzers (Brief)Werk. In E. Decultot, P. Kampa und J. Kittelmann (Hg.), Johann Georg Sulzer: Aufklärung im Umbruch (S. 252-285). Berlin und Boston: de Gruyter. 
Klopstock, F. G. (1979). Werke und Briefe. Abt. B, Bd. 1: Briefe 1738-1750. Hg. v. Horst Gronemeyer. Berlin und New York: de Gruyter.

Knebel, K., Maul, G., \& Schmuck, T. (Hg.). (2019). Abenteuer der Vernunft. Goethe und die Naturwissenschaften um 1800. Ausstellungskatalog. Dresden: Sandstein.

Humboldt, A. v., Kohlrausch, H. (2019). Die Kosmos-Vorlesung an der Berliner Sing-Akademie (C. Kassung und C. Thomas, Hg.). Berlin: Insel.

Lavater, J. C. (1775). Physiognomische Fragmente, zur Beförderung der Menschenkenntniß und Menschenliebe. Bd. 1. Leipzig: Weidmanns Erben und Reich.

Ledermüller, M. F. (1762). Nachleese seiner mikroskopischen Gemüths- und Augen-Ergötzung; I. Sammlung. Bestehend in zehen fein illuminirten Kupfertafeln, sammt deren Erklärung: und einer getreuen Anweisung, wie man alle Arten Mikroskope, geschickt, leicht und nüzlich gebrauchen solle. Verlegt und in Kupfer gebracht von Adam Wolfgang Winterschmidt. Nürnberg: de Launoy.

Lepenies, W. (1988). Autoren und Wissenschaftler im 18. Jahrhundert. Buffon, Linné, Winckelmann, Georg Forster, Erasmus Darwin. München et al.: Hanser.

Lesser, F. C. (1738). Insecto-Theologia, oder: vernunff- und schrifftmäßiger Versuch, wie ein Mensch durch aufmercksame Betrachtung derer sonst wenig geachteten Insecten zu lebendiger Erkänntniß und Bewunderung der Allmacht, Weißheit, der Güte und Gerechtigkeit des grossen Gottes gelangen könne. Frankfurt am Main und Leipzig: Blochberger.

Lütteken, L. (Hg.). (2003). Urbanität als Aufklärung. Karl Wilhelm Ramler und die Kultur des 18. Jahrhunderts. Göttingen: Wallstein.

Michler, W. (2013). Klassifikation und Naturform. Zur Konstitution einer Biopoetik der Gattungen im 18. Jahrhundert. In M. Bies, M. Gamper und I. Kleeberg (Hg.), Gattungs-Wissen. Wissenspoetologie und literarische Form (S. 35-50). Göttingen: Wallstein.

Mödersheim, S. (1995). „Auch die fruchtbarsten Bäume wollen beschnitten sein“: Georg Friedrich Meiers Konzept der Einbildungskraft und Dichtungskraft und die Kritik an Anna Louisa Karsch. In T. Verweyen \& H.-J. Kertscher (Hg.), Dichtungstheorien der deutschen Frühaufklärung (S. 37-54). Tübingen: Niemeyer.

Mylius, C. (1753). Nicht kursiv Reise auf den Blocksberg. Physikalische Belustigungen, 1753, 1023-1029.

Ramler, K. W. (1767). Oden. Berlin: Voß.

Richter, K. (1972). Literatur und Naturwissenschaft. Eine Studie zur Lyrik der Aufklärung. München: Fink.

Schüddekopf, C. (Hg.). (1899). Briefwechsel zwischen Gleim und Uz. Tübingen: Litterar. Verein.

Schüddekopf, C. (Hg.) (1906/1907). Briefwechsel zwischen Gleim und Ramler. 2 Bde. Tübingen: Litterar. Verein.

Segeberg, H. (1997). Literatur im technischen Zeitalter. Von der Frühzeit der deutschen Aufklärung bis zum Beginn des Ersten Weltkrieges. Darmstadt: Wiss. Buchgesellschaft.

Shteir, A. B. (1976/1977). Albrecht von Haller's Botany and „Die Alpen“. Eighteenth-Century Studies, 10(2), 169-184.

Stadler, U. (2003). Der technisierte Blick: Optische Instrumente und der Status von Literatur. Ein kulturhistorisches Museum. Würzburg: Königshausen \& Neumann.

Stört, D. (2018). Das „Samen-Cabinet“ von Christian Reichart (1685-1775). Ästhetische und epistemische Funktionen von Samenschränken im 18. Jahrhundert. In J. Kittelmann (Hg.), Botanik und Ästhetik (S. 171-185). Göttingen: Universitätsverlag.

Stuber, M. (2005). Hallers Netz. Ein europäischer Gelehrtenbriefwechsel zur Zeit der Aufklärung. Basel: Schwabe.

Sukopp, H. (2011). Gleditschs Experimentum berolinense aus den Jahren 1749-1751. Verhandlungen des botanischen Vereins Berlin-Brandenburg, 144, 45-61.

Sulzer, J. G. (1745). Versuch einiger Moralischen Betrachtungen über die Werke der Natur. Nebst einer Vorrede von A. F. W. Sack. Berlin: Haude.

Sulzer, J. G. (1746). Vorrede des Herausgebers. In J. J. Scheuchzer, Natur-Geschichte des Schweitzerlandes. Samt seinen Reisen über die Schweitzerische Gebürge (S. I-VIII). Zürich: Gessner.

Sulzer, J. G. (1747). Beschreibung der Merckwürdigkeiten, welche er in einer Ao. 1742. gemachten BergReise durch einige Oerter des Schweitzerlandes beobachtet hat. Zürich: Gessner.

Sulzer, J. G. (1750). Unterredungen über die Schönheit der Natur. Berlin: Haude und Spener.

Sulzer, J. G. (1758). Description d'un barometre portatif, avec une nouvelle méthode pour faire des thermometres d'une division constante. Acta Helvetica, physico-mathematico-anatomico-botanico-medica, 3, 259-265. 
Sulzer, J. G. (1771). Allgemeine Theorie der schönen Künste. Bd. 1. Leipzig: Weidmanns Erben und Reich.

Wieland, C. M. (1752). Die Natur der Dinge in sechs Büchern. Mit einer Vorrede Georg Friedrich Meiers. Halle: Hemmerde.

Wolf, B. (2015). Räume des Wissens. In J. Dünne \& A. Mahler (Hg.), Handbuch Literatur und Raum (S. 115-125). Berlin und Boston: de Gruyter.

Zimmermann, J. G. (1756). Betrachtungen über die Einsamkeit. Zürich: Heidegger und Compagnie.

Publisher's Note Springer Nature remains neutral with regard to jurisdictional claims in published maps and institutional affiliations. 